example illustrates that epidemiologic data need to be collected to inform clinical trials and decision-making for health practice. As single cohort studies are carried out around the world, we can begin to synthesize the incomplete epidemiologic knowledge base for use in policy and practice. These reviews will also uncover gaps in our knowledge base that can be filled by new research from ongoing studies.

It is time that we develop a global public health genomics initiative that builds on the currently fragmented efforts of geneticepidemiologic research around the world. This initiative can be developed through public-private-academic collaborations. In particular, we need to build a robust process that allows data from many biobanks to be integrated through standardized platforms for joint analyses. Also, we need to integrate data obtained from all valid epidemiologic study designs, notably population-based incident casecontrol studies. Systematic synthesis of epidemiologic data takes time and skills and should be allocated sufficient resources. This proposed initiative can take us a long way towards translating human genome discoveries into population health benefits for citizens of the twenty-first century.

\section{Muin J Khoury}

Office of Genomics and Disease Prevention, Centers for Disease Control and Prevention, 1600
Clifton Road, Mailstop E82, Atlanta, Georgia 30333, USA. Correspondence should be addressed to M.J.K. (mkhoury@cdc.gov).

1. Collins, F.S. Nature $429,475-477$ (2004).

2. Khoury, M.J., Millikan, R., Little, J. \& Gwinn, M. Int. J. Epidemiol. (in the press).

3. Khoury, M.J., Beaty, T.H. \& Cohen, B.H. Fundamentals of Genetic Epidemiology (Oxford University Press, New York, 1993).

4. Thomas, D.C. Statistical issues in the design and analysis of gene-disease association studies. in Human Genome Epidemiology: A Scientific Foundation for Using Genetic Information to Improve Health and Prevent Disease (eds. Khoury, M.J., Little, J. \& Burke, W.) 92-110 (Oxford University Press, New York, 2004).

5. Vanddenbroucke, J.P. et al. Lancet 344, 1453-1457 (1994).

6. Sass, A.E. \& Neufeld, E.J. Curr. Opin. Pediatr. 14, 370-378 (2002).

7. Khoury, M.J., McCabe, L.L. \& McCabe, E.R. N. Engl. J. Med. 348, 50-58 (2003).

\title{
Phylogenetic validation of horizontal gene transfer?
}

\section{To the editor:}

The study by Nakamura and coworkers ${ }^{1}$ offers insight into the computational analysis of horizontal gene transfer. Their results seem to be convincingly supported by phylogenetic validation of the supplied examples of calculated horizontal gene transfer events. An outstanding example, validating their method, concerns the presence of the gene encoding an rRNA adenine N-6-methyltransferase, NMB0066, in the genome of Neisseria meningitidis MC58 (ref. 2). According to the authors' results, NMB0066 originates from plasmids naturally occurring in Staphylococcus aureus, such as pE5. In fact, this gene, being an erythromycin resistance cassette (ermC), was horizontally acquired, because it was deliberately introduced in the $N$. meningitidis MC58 genome by genetic modification using plasmid pIP10 (ref. 3) to reduce virulence. In the pIP10 construct, the gene encoding the polysialic acid capsule biosynthesis protein $\mathrm{SiaD}$ (NMB0067) is inactivated by insertion of cloning vector sequences and the erm $C$ gene originally derived from plasmid pIM13, a naturally occurring plasmid found in Bacillus subtilis ${ }^{4}$. Remnants of cloning vector sequences flanking NMB0066 are noticeable in the genome sequence of N. meningitidis MC58. The sequences of NMB0066 and erm C of pIM13 are identical, whereas that of erm $C$ of pE5 contains one nonsynonymous mutation and an insertion of 107 nucleotides upstream of the open reading frame. This means that, although NMB0066 is clearly horizontally acquired by N. meningitidis MC58, its origin remains at best obscure. In addition, it is implausible that the surrounding genes, NMB0065 through NMB0070, were acquired in one event from the same donor as erm $C$, opposing the authors' suggestion that they were transferred simultaneously with NMB0066. In conclusion, although the algorithm by Nakamura and coworkers correctly identified the acquisition of NMB0066 by $N$. meningitidis, their suggestion that $S$. aureus was the donor organism is improbable. Moreover, their interpretation concerning the simultaneous acquisition of NMB0066 and its surrounding genes is inappropriate.

\section{Mark van Passel, Aldert Bart, Yvonne}

Pannekoek \& Arie van der Ende

Academic Medical Center, Department of Medical Microbiology, University of Amsterdam, Amsterdam, the Netherlands. Correspondence should be addressed to A.v.d.E.

(a.vanderende@amc.uva.nl).

1. Nakamura, Y., Itoh T., Matsuda, H. \& Gojobori, T. Nat. Genet. 36, 760-766 (2004).

2. Tettelin, H. et al. Science 287, 1809-1815 (2000).

3. Virji, M. et al. Mol. Microbiol. 18, 741-754 (1995).

4. Monod, M., Denoya, C. \& Dubnau, D. J. Bacteriol. 167, 138-147 (1986).

\section{The use of genome annotation data and its impact on biological conclusions}

To the editor:

We were interested to read the recent paper by Nakamura et al. ${ }^{1}$ describing a new technique to identify horizontally acquired genes in bacterial genomes. But we were surprised to see that NMB0066, a gene from the Neisseria meningitidis MC58 genome, was used as an example of horizontal transfer. In fact, NMB0066 is part of an artificial erythromycin resistance cassette that was inserted into the capsule gene siaD (NMB0067) to disrupt it, rendering the MC58 strain less virulent and therefore less hazardous to manipulate in the laboratory. The annotation of NMB0066 submitted to the public databases clearly indicates that it is foreign: "NMB0066 rRNA adenine N-6-methyltransferase (ErmC); foreign cassette inserted to disrupt NMB0067 
(SiaD) to reduce virulence." This gene may be a good positive control for the in silico approach used by Nakamura et al., but it is biologically irrelevant in the context of this genome. Therefore, the further discussion on how this gene and the capsule locus in which it is inserted were horizontally transferred from Staphylococcus aureus is meaningless. The capsule locus probably was recently acquired by MC58 but probably not directly from $S$. aureus.

This example highlights the importance of not taking the output of any bioinformatics program at face value; the results should always be interpreted in the biological context of the organism or sequence under study, and the relevant literature should be thoroughly examined. In this case, however, reading the literature may not have helped; in the original sequence paper ${ }^{2}$ the gene NMB0066 was mentioned as being within an island of horizontal transfer (the capsule locus), but, due to an oversight, the specific reason for its presence was not spelled out. Notwithstanding the fact that this was described in the annotation submitted to the public databases, this may have been misleading for the casual reader, for which we apologize. This serves to underscore the need to be rigorous in interpreting data, both one's own and those from other groups.

\section{Hervé Tettelin ${ }^{1}$ \& Julian Parkhill ${ }^{2}$}

${ }^{1}$ The Institute for Genomic Research, 9712 Medical Center Drive, Rockville, Maryland 20850, USA. ${ }^{2}$ The Sanger Institute, Wellcome Trust Genome Campus, Hinxton, Cambridge, CB10 1SA, UK. Correspondence should be addressed to H.T. (Tettelin@tigr.org).

1. Nakamura, Y., Itoh, T., Matsuda, H. \& Gojobori, T. Nat. Genet. 36, 760-766 (2004).

2. Tettelin, H. et al. Science 287, 1809-1815 (2000). 